\title{
RECONFIGURACIÓN REGIONAL EN EL MARCO DEL ORDENAMIENTO TERRITORIAL; FRANCIA, UN ESPEJO PARA COLOMBIA
}

\section{REGIONAL RECONFIGURATION IN THE CONTEXT OF LAND-USE PLANNING; FRANCE, A MIRROR FOR COLOMBIA}

\author{
Adriana Posada Arrubla ${ }^{1}$, Ana Elena Berrocal Mendoza ${ }^{2}$ \\ ${ }^{1}$ Economista Agrícola, Esp. Gestión Social y Ambiental, M.Sc. Planeación Urbana y Regional, Docente Facultad de Ing- \\ enierías U.D.C.A, calle 222 \#55-37, Bogotá D.C., Colombia, e-mail: aposada@udca.edu.co; ${ }^{2}$ Ingeniera Ambiental, Esp. \\ Gerencia Ambiental, M.Sc. Políticas y Desarrollo Sostenible, Institut Catholique de Paris-Francia, 3 Rue Davioud 75016, \\ e-mail: anaberrocal19@gmail.com
}

Rev. U.D.C.A Act \& Div. Cient. 18(1): 271-281, Enero-Junio, 2015

\section{RESUMEN}

En el proceso de ordenamiento territorial de Colombia, se espera una nueva configuración del país, lo cual, crea la expectativa de una reconfiguración basada en regiones; sin embargo, este esquema de ordenamiento territorial resurge desde una óptica administrativa, lo que, a su vez, genera dudas sobre su relación con varios asuntos: la descentralización, el modelo de desarrollo territorial nacional, la asociatividad y los proyectos colectivos con carácter regional. Para aportar al entendimiento de esta cuestión, se presenta en este artículo, la experiencia regional de Francia, como referente mundial y como un espejo para analizar la situación colombiana.

Palabras clave: Regionalización Colombia, regionalización Francia, desarrollo territorial.

\section{SUMMARY}

In the process of land use planning of Colombia is expected to a new configuration of the country, which creates the expectation of a regions-based reconfiguration; However, this zoning scheme resurfaces from an administrative perspective, which in turn raises questions about his relationship with several issues: decentralization, the national territorial development model, the associativity and collective projects on a regional basis. To contribute to the understanding of this issue, is presented in this article the regional experience of France as a world leader and as a mirror to analyze the situation in Colombia.

Key words: Regionalization Colombia, regionalization France, territorial development.

\section{INTRODUCCIÓN}

El ordenamiento territorial es un proceso que utiliza diversas estrategias e instrumentos para lograr una relación armónica población-territorio. Una de tales estrategias es la conformación o configuración de regiones, entendidas como espacios territoriales con dinámicas propias, que generan funcionalidad para tal espacio. Para Boiser (1998), la región es lo más próximo a la Nación y sirve para funciones más complejas, que las de su escala inmediatamente menor. Según Reyes \& Córdoba (2012), la región es una referencia espacio-territorial, lo que obliga a comprender el poder de manera multiescalar.

Por otro lado, Santos (2000) reconoce la región como un espacio de conveniencia, con un nivel de complejidad difícil de detectar. Por su parte, Pinzón \& Echeverri (2012) establecen que la dinámica territorial de una región es su transformación material o intangible en función de las potencialidades y restricciones ambientales asociadas al territorio y como respuesta a modos de influencia y presión, que ejercen sobre éste los actores sociales. Según Espejo (2003), una misma extensión de territorio puede ser divido en regiones naturales, culturales, económicas o urbanas, entre otras opciones, pero a la regionalización le da sentido el objetivo trazado; por ello, el concepto de región ha evolucionado pasando por los enfoques natural, geográfico, funcional, sistémico, económico, urbanístico.

Actualmente, en Colombia, se confunde la regionalización, debido a que sigue siendo un tema sin agotar y se une a otro que tiene como factor común, varias interpretaciones: el proceso de ordenamiento territorial. Para Cuervo (2003), un asunto son las regiones y, otro, la regionalización de las 
políticas públicas. Para analizar el ordenamiento territorial de Colombia, desde la posible reconfiguración regional, resulta conveniente hacer una comparación frente a un país con reconocido trasegar histórico en la administración regional de su territorio, como lo es Francia. La geografía francesa fue la primera en el mundo, en desarrollar una manera específica de realizar estudios regionales, que intentaban interpretar las regiones, a partir de la relación de sus habitantes con su entorno (Reyes \& Córdoba, 2012).

Respecto a la situación mundial, el IMC (2014), explica que la redefinición de los límites franceses territoriales internos, al pasar de 22 a 13 regiones (Mapa 1), es una tendencia internacional para simplificar la administración del territorio y reducir costos de gestión pública. Y asegura que, recientemente, varios países europeos han reducido el número de regiones, de provincias y de municipios: Grecia, pasó de 54 a 13 provincias; Dinamarca, de 14 a 5 regiones y Suecia, reducirá sus 21 regiones, a menos de la mitad. En general, la Unión Europea representa una organización de estados prácticamente única, en la que son esenciales las regiones, para la coordinación entre los diferentes niveles territoriales (Mendoza et al. 2013).

La parlamentaria europea Rodrigues (2010), señala que la Unión Europea, al tiempo que implementa su nuevo marco institucional (según Tratado de Lisboa), tiene dos desafíos: definir su papel en el nuevo orden internacional y renovar su modelo de desarrollo (Estrategia EU 2020). Este escenario de desafíos globales requiere mecanismos de gobernanza multiescalares, sobre los cuales, Miedes \& Sánchez (2014) alertan de la existencia de tres grandes bloques de tensiones entre:

- Producción y consumo us. disponibilidad de recursos no renovables;

- Fuerzas centrípetas (creciente interdependencia económica) us. fuerzas centrífugas (creciente diferenciación territorial);

- Acelerada urbanización vs. fragmentación y exclusión urbana.

En escalas y contextos diferentes, en Francia y en Colombia, se presentan estas tensiones, por que atraviesan momentos que exigen un claro modelo de desarrollo, en el marco del ordenamiento territorial. Francia, con más de 30 años en su proceso de regionalización y de descentralización, dirige desde hace cuatro años, una reforma territorial para fortalecer el poder regional. Colombia adoptó por primera vez, hace cuatro años, una Ley Orgánica de Ordenamiento Territorial (LOOT 1451/2011), impulsando la cooperación intermunicipal, la creación de regiones administrativas y de planificación (RAP) y de regiones de gestión y de planificación (RGP). Para Carrizosa et al. (2002), aun así, Colombia sigue en recurrente dicotomía sobre la región, el Estado federal y el centralis- mo, mientras desprecia la posibilidad de formas viables que actúen en las interfases.

Desde 1998, el CIEP reconocía que, a pesar de comprenderse las dinámicas regionales y su articulación con los procesos nacionales e internacionales, existía incertidumbre por el objeto de la región. Hoy, la situación es igual, con algunos cambios en la perspectiva o el enfoque. Por su parte, Fals Borda (1993) describe cómo desde 1930 renacieron juntos los temas de región y de provincia, en una coyuntura de factores sociales y geográficos del Caribe (la depresión Momposina), donde se sentía la orfandad administrativa. Señala, que desde 1960, la reflexión sobre la regionalización se profundizó y, hoy, con la vigente Constitución Política Colombiana de 1991, se creó una Comisión de Ordenamiento Territorial, para dar nuevas luces al asunto.

20 años después, Fals Borda (2000) reflexionaba a partir de lo ocurrido en Francia y estimaba que se necesitarán los esfuerzos de varias generaciones para llegar a un nuevo mapa de Colombia, con su dinámica realidad y con factores de unidad y progreso colectivo. En el mapa 2, se puede apreciar la subdivisión de Colombia, del Instituto Geográfico Agustín Codazzi, en seis regiones geográficas (antes regiones naturales); el mapa de regiones administrativas no existe, debido a que no están aún definidas, asunto que continúa en proceso como una propuesta, entre varias que han existido, desde diferentes enfoques de la regionalización (Sánchez Valbuena, 2004). Con el fin de profundizar sobre la reconfiguración regional en Colombia, en el marco del proceso de ordenamiento territorial, se realiza este artículo, en el que se aportan algunos elementos de análisis.

\section{MATERIALES Y MÉTODOS}

Para identificar y comparar posibilidades de reconfiguración regional en el marco del ordenamiento territorial de dos países, se previó que uno fuera un importante referente mundial en el tema. Francia, como espejo de Colombia, en donde, desde una posición político-administrativa, se ha originado expectativa sobre una nueva reconfiguración regional. Y aunque los dos territorios tienen marcadas diferencias entre sí, la comparación se hace sobre los instrumentos con que cuentan las autoridades e instancias territoriales y la manera de hacerlos funcionar, para lograr desarrollo en su propio contexto. Desde la perspectiva económica, el FMI (2014) ha clasificado a los países en dos grupos: los avanzados (36 países, donde Francia hace parte de la zona del euro) y los emergentes en desarrollo (153 Naciones, donde Colombia hace parte de la región América Latina y el Caribe), extremos que ayudan a identificar el porqué del ejercicio comparativo.

En primera instancia, con base en información secundaria obtenida en cada país, se describe la manera cómo funcio- 


\section{La carte des 13 régions adoptée par l'Assemblée nationale}

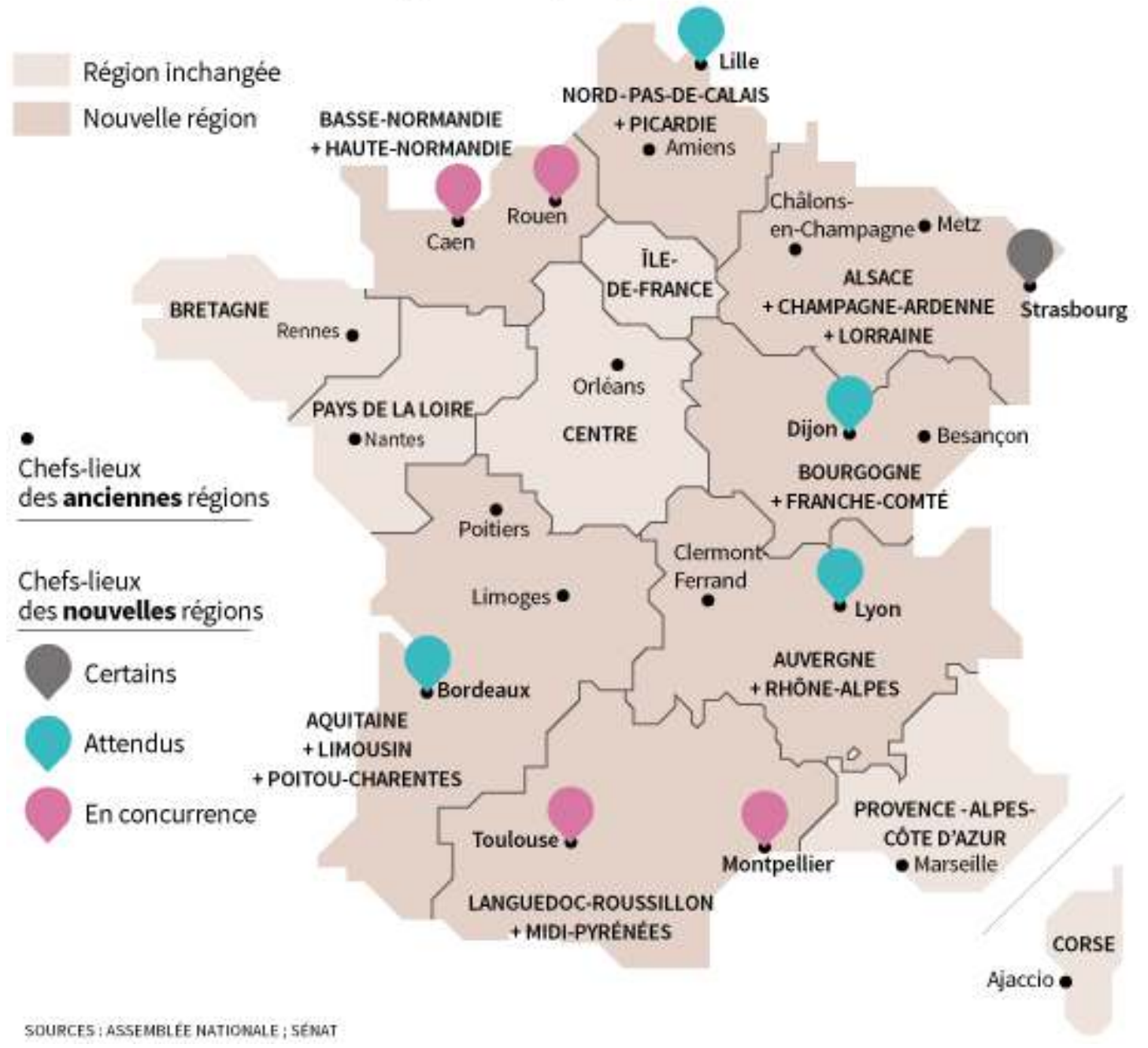

Mapa 1. Antiguas y nuevas regiones de Francia. 2014. 


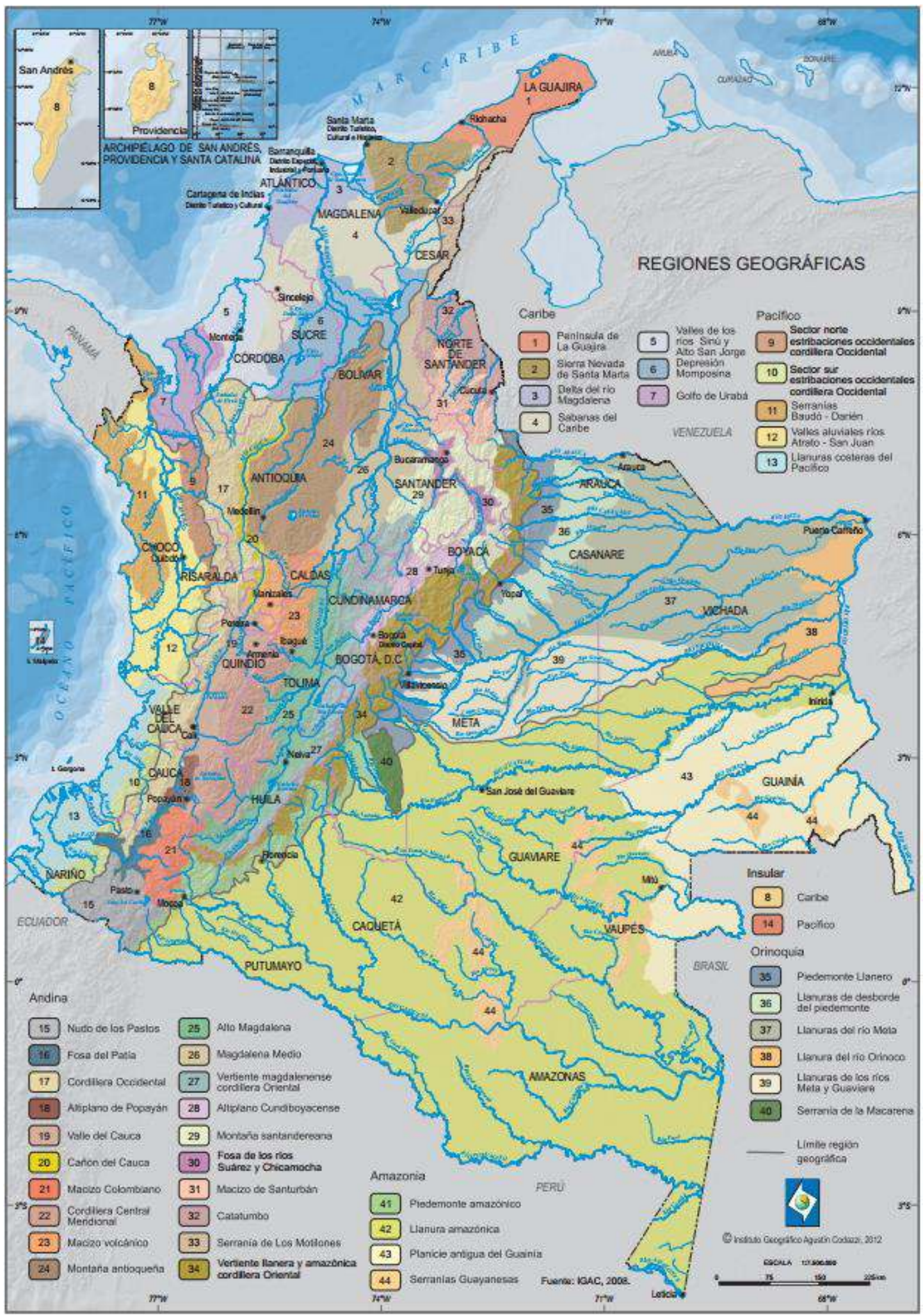

Mapa 2. Regiones geográficas (naturales) de Colombia. IGAC, 2012. 
nan las entidades territoriales y la forma cómo se han presentado los procesos asociativos en Colombia y en Francia, para posteriormente describir el movimiento actual de optimización territorial en Francia. Con los datos de estos insumos, se entrevistaron a varios personajes claves en Colombia, que han tenido un rol importante en las iniciativas de regionalización, lo que permite concluir sobre las lecciones que Colombia puede extraer de la reforma territorial francesa en curso, para avanzar en sus propias decisiones, de acuerdo con su particular sistema de desarrollo.

\section{RESULTADOS Y DISCUSIÓN}

En América Latina, el proceso descentralización profundizó la complejidad de la planificación y el ordenamiento territorial, dado que la articulación de actores y sectores requiere mayores esfuerzos, para alinear los niveles del Estado y coordinar su administración (CEPAL, 2014). El asunto de la planeación y el ordenamiento territorial en Colombia está sustentado desde normas orgánicas, referidas a la planeación de entidades territoriales, a los municipios y a las áreas metropolitanas, pero no a las regiones, ni a los departamentos, lo cual, genera inquietud sobre el peso que tienen estos referentes entre sí, pues a pesar que departamentos y regiones tienen un área espacial mayor, son inferiores en la preponderancia administrativa del país.

Como se observa en el cuadro 1, los aspectos relacionados con el ordenamiento territorial desde las regiones, para Colombia y para Francia, contienen diferenciadas situaciones. En Colombia, las competencias para los 1.102 municipios exigen mucha solidez, como el principal ente territorial, capaz de dinamizar el desarrollo supramunicipal, garantizando: concordancia con planes de desarrollo departamental y de resguardos indígenas; alianzas y sinergias público-privadas y en zonas fronterizas, con herramientas de integración para el desarrollo sostenible; gestión de los recursos naturales; sostenimiento de la industria nacional, el comercio y el consumo.

En Francia, son las regiones quienes tienen competencia principal para gestionar el suelo urbano (plan local de urbanismo equivalente en Colombia al plan de ordenamiento territorial municipal) y autonomía para reglamentar el urbanismo, pero respetando la concertación y las prescripciones nacionales (Grandguillot, 2014). Son 21 regiones más la entidad territorial de la isla de Corsega, es decir, 22 regiones francesas; con la ley del 27/2002 varias competencias del Estado fueron transferidas a las regiones (el plan regional de calidad del aire y la clasificación de reservas naturales regionales). Más adelante, con la ley del 13/2004, sobre libertades y responsabilidades locales, se consolida como la entidad territorial con mayor transferencia de competencias.
Los procesos asociativos en Colombia y Francia: En los últimos años en Colombia, se han introducido instrumentos normativos que privilegian la asociación territorial para generar sinergia, complementariedad, competencia y equidad territorial. Leyes, como la 152/1994, la LOOT y la ley 1530 de 2012 del sistema general de regalías, favorecen la creación de esquemas asociativos territoriales, que tienen por objetivo el desarrollo regional. Los esquemas asociativos aparecen, entonces, como herramientas de articulación y de desarrollo territorial, capaces de abordar los problemas y las temáticas que no pueden ser solucionadas, efectivamente, de manera individual, por los municipios y los departamentos.

La LOOT concibe la asociación como una herramienta que permite la puesta en marcha de proyectos, de programas y de acciones de desarrollo de interés común entre las entidades territoriales, privilegiando la cooperación territorial, para generar más competitividad e inversión pública. En su artículo 9 posibilita la constitución de: asociaciones de entidades territoriales (municipios, departamentos, áreas metropolitanas), metrópolis, provincias administrativas y de planificación, regiones administrativas y de planificación (RAP), regiones de planificación y de gestión (RPG), asociaciones de corporaciones autónomas regionales, alianzas estratégicas para el desarrollo económico con países fronterizos y alianzas estratégicas de orden económico. Para desarrollar la RAP y la RPG establece que serán espacios para la inversión, el desarrollo y la competitividad y para convertirse en entidades territoriales, deberán iniciar una etapa de preparación, constituyendo una de tales figuras. Paradójicamente, la LOOT no le otorga a la región opción para recibir directamente transferencias de recursos económicos, ni administrarlos.

Según el DNP (2013a), se han generado cinco procesos de asociación departamental y 49 procesos de asociación intercomunal; de ellos, 42 bajo la forma de asociación de municipios, cuyas principales dificultades se relacionan con la ausencia de fondos de financiamiento, que garanticen la perennidad y operatividad de las asociaciones. Se destaca la creación de la RAPE central, conformada por cinco entidades territoriales, que buscando economías de escala, para resolver problemas públicos y según la AMB (2014), una de sus tareas es gestionar su reconocimiento en el esquema de inversión nacional. El DNP (2013b) destaca que en los procesos de reciente surgimiento, parte de sus motivaciones, yace en la posibilidad de financiar proyectos de carácter regional, a través del sistema general de regalías y de los contratos-plan. De hecho, se han iniciado procesos de desarrollo integral, mediante la formulación de planes y de proyectos estratégicos, con visión de largo plazo, apoyados en algunos casos por la Nación.

En el caso de Francia, la cooperación intercomunal apareció hace más de 120 años (ley del 22 de marzo de 1890), dife- 
Cuadro 1. Aspectos sobre ordenamiento regional en Colombia y Francia.

\begin{tabular}{|c|c|c|}
\hline ASPECTO & EN COLOMBIA & EN FRANCIA \\
\hline $\begin{array}{l}\text { Proceso de } \\
\text { regionalización }\end{array}$ & $\begin{array}{l}\text { Inicia en } 1960 \text {, pero no alcanza a } \\
\text { concretarse }\end{array}$ & $\begin{array}{l}\text { Inicia en } 1960 \text { y se mantiene. Ha tenido varias fases y } \\
\text { ahora se refuerza }\end{array}$ \\
\hline Normativa & $\begin{array}{l}\text { Constitución Política (1991) } \\
\text { Ley orgánica Plan desarrollo (1994) } \\
\text { Ley orgánica ordenamiento territorial } \\
\text { (2011) }\end{array}$ & $\begin{array}{l}\text { Ley de ordenación y desarrollo sostenible del territorio } \\
\text { (1999) } \\
\text { Ley de solidaridad y renovación urbana (2000) }\end{array}$ \\
\hline $\begin{array}{l}\text { Plan nacional de } \\
\text { Ordenamiento } \\
\text { Territorial }\end{array}$ & No existe aún & Existe desde 1950 y con carácter binacional \\
\hline Municipios & $\begin{array}{l}\text { Régimen legislativo definido que establece } \\
\text { claramente sus competencias y son } \\
\text { las principales entidades en materia de } \\
\text { ordenamiento territorial. Son \#1102 (en } \\
\text { 2013) }\end{array}$ & $\begin{array}{l}\text { Entidad administrativa de base; es las más antigua } \\
\text { y familiar para los administradores políticos; fueron } \\
\text { creados a través de la ley del 14/1789. Son \#36.767 } \\
\text { municipios (en 2013) }\end{array}$ \\
\hline Departamentos & $\begin{array}{l}\text { Se vienen debilitado y no tienen normativa } \\
\text { actualizada sobre sus competencias }\end{array}$ & $\begin{array}{l}\text { Son objeto de tentativas constantes de supresión, como } \\
\text { es el caso de la nueva reforma territorial. Son \# } 96 \\
\text { departamentos en el territorio continental y } 3 \text { más en } \\
\text { ultramar. }\end{array}$ \\
\hline Regiones & $\begin{array}{l}\text { No tienen reglamentación sobre } \\
\text { competencias o asignación de recursos, } \\
\text { pero la nueva LOOT establece una base } \\
\text { legal frente a su eventual creación }\end{array}$ & $\begin{array}{l}\text { División administrativa, tanto entidad territorial } \\
\text { descentralizada como circunscripción administrativa } \\
\text { del Estado; es a la vez, la más grande entidad territorial } \\
\text { de derechos y la más reciente. Con la ley del } 2 / 1982 \text {, } \\
\text { se le asignaron competencias en el desarrollo, } \\
\text { el ordenamiento territorial, la planificación y el } \\
\text { equipamiento, destacándose la gestión con empresas, } \\
\text { de los contratos-plan y del transporte }\end{array}$ \\
\hline $\begin{array}{l}\text { Áreas } \\
\text { Metropolitanas }\end{array}$ & Ley orgánica 1625/2013 & Proyecto y contrato de aglomeración \\
\hline
\end{tabular}

rentes leyes sucesivas la han reforzado, entre ellas la reforma territorial de 2010 y la reforma actual. Es así, como el desarrollo intermunicipal da lugar a recomposiciones territoriales y gubernamentales y, por ello, los representantes de los municipios enfrentan el ejercicio complejo de la cooperación y la definición de todo un proyecto territorial (Loubet, 2013). Una forma de cooperación corresponde a una intermunicipalidad de gestión y, la otra, a una intermunicipalidad de proyectos, para las cuales, la financiación o la transferencia de recursos, es diferente. La primera es sin fiscalidad propia, pues depende de la contribución de los municipios miembros, donde la cuota es un principio establecido en los estatutos del establecimiento. La segunda está sujeta a un régimen fiscal, lo que permite a los municipios disponer de ingresos fiscales directos. (Grandguillot, 2014).

Existen, además, los establecimientos públicos de cooperación intermunicipal (fondement des EPCI) y son distintos a los municipios, de los que hacen parte. Esta cooperación, se fundamenta en su libre voluntad para elaborar proyectos comunes de desarrollo, en el marco de perímetros de solidaridad. Desde la adopción de la reforma territorial de 2010, son establecimientos públicos de cooperación intercomunal los siguientes (art. L.5210-1-1 du CGCT): sindicatos de municipios, comunidades de municipios, las comunidades urbanas, las comunidades de aglomeración, los sindicatos de nuevas aglomeraciones y las metrópolis. Entre los $\mathrm{EPCl}$ y los municipios existe una lógica de solidaridad, de interés comunitario, que constituye la línea del compartir entre el municipio y las asociaciones de municipios.

Adicionalmente, se destaca el esquema regional de ordenamiento y desarrollo sostenible del territorio (SRADDT -sigla en idioma francés-), documento con orientaciones estratégicas sobre ordenamiento territorial, para que las regiones alcancen un desarrollo sostenible y que el Estado genere una coherencia territorial, transmitiendo sus objetivos nacionales a la escala regional. Son una herramienta indispensable de 
planificación del territorio francés, por la responsabilidad en la coherencia de los proyectos relativos a las políticas nacionales, en materia de ordenamiento territorial (Arches et al. 2013). La región tiene así la oportunidad de desarrollar su territorio, ya que cada una establece un desafío principal por realizar y constituye el aspecto diferenciador con los demás SRADDT, de las otras regiones (Dotsey, 2013).

El movimiento actual de optimización territorial en Francia: La ley de descentralización de 1982 marcó una etapa importante en el proceso de regionalización francés, al reconocer, por primera vez, a las regiones como entidades territoriales. Según Pasquier (2012), los presupuestos regionales han aumentado el gasto público general del país, hecho que ha suscitado, desde hace más de 25 años, por parte de los gobiernos de derecha e izquierda, la apuesta por diferentes reformas territoriales, tendientes a reducir la parte del impuesto local en los ingresos de las entidades territoriales. El gobierno, buscando eficiencia, conduce desde el 2008, una reforma estructural de la organización territorial, que es objeto de dos proyectos de ley (presentados al consejo de ministros el 18 junio/2014): un proyecto reconfigura la carta de regiones y, el otro, precisa las competencias de las regiones, tendientes a reforzar su potencial y a apoyar aún más la intermunicipalidad.

La reforma territorial busca una reducción anual de 12 a 25 millares de euros en el presupuesto de las entidades territoriales que, actualmente, es de 250 millares de euros. Esta reducción presupuestal será el resultado de la fusión de regiones, el reagrupamiento de intermunicipalidades y la supresión de los consejos generales (equivalente a gobernaciones en Colombia). El secretario de Estado, estima que las políticas nacionales no serán eficaces si las regiones no tienen la fuerza necesaria para competir internacionalmente; esto significa, un tamaño geográfico y un peso geográfico mucho más importante que el actual; las de bajo peso geográfico son las que cuentan únicamente con dos o tres departamentos (LNRF, 2014). El proyecto de ley que precisa las regiones fue presentado a la asamblea nacional en mayo/2014; los diputados tienen tiempo hasta junio de 2015, para hacer las propuestas respectivas con relación a las posibles fusiones de regiones, pero el 23 de julio/2014, una parte de la reforma territorial referente a la nueva carta de regiones fue aprobada, reduciendo el número de regiones, que pasó de 22 a 13.

A futuro, Francia planea proponer al 2016, una nueva carta geográfica definitiva de regiones y el próximo objetivo del gobierno, en materia de reforma territorial, es de incentivar la intermunicipalidad, hacerlas crecer en poder y de privilegiar la transferencia de competencias de los municipios, a estas formas de asociación. Otro elemento clave de la reforma territorial en curso es la de los consejos generales (Goberna- ciones en Colombia), estos tienden a desaparecer, debido a la creación de grandes regiones con mayores competencias y del fortalecimiento de la intermunicipalidad (asociación de municipios). Para acompañar esta transición, una revisión constitucional, previendo la supresión de los consejos regionales en 2020, será concebida por el gobierno.

Con lo anterior, se establece que el asunto de la reconfiguración regional en Colombia no es tanto de tipo espacial, es más de corte político, de enfoque sobre el modelo de desarrollo territorial y la regionalización misma y, sobre todo, de interés por asuntos estratégicos colectivos. Al profundizar en la regionalización colombiana, se detecta que ésta ha resurgido desde una forma de interpretar el ordenamiento territorial como un hecho administrativo. La discusión gira en torno a cuatro temas que de aquí se derivan: la relación entre reconfiguración regional y descentralización; la necesidad de un modelo de desarrollo territorial nacional; la asociatividad como estrategia optimizadora de recursos y los proyectos colectivos con carácter regional.

Por una parte, un requisito fundamental para que funcione la región como entidad territorial, con competencias precisas y buenos recursos financieros, es que exista una verdadera descentralización; en Colombia, se inició el proceso, pero a la fecha los resultados son mínimos. Por otra, es necesario que exista un claro modelo de desarrollo definido en conjunto con la sociedad, para poder establecer si al interior de tal modelo, la regionalización ayuda a concretarlo; en Colombia, no existe un modelo de desarrollo y, mucho menos, en el marco de un proceso de ordenamiento territorial. Según Cuervo (2003) es inevitable mirar la región y el ordenamiento territorial a la luz de los retos y de las dificultades creadas a partir de la descentralización; los temas de mayor importancia son la gobernabilidad, la autonomía fiscal, la sustentabilidad económica, la eficiencia administrativa y la autonomía local.

Ramírez (2012) detectó una ausencia de convergencia regional en Colombia y la profundización de la desigualdad en grupos de regiones, que tienden a rezagarse. Según Espinosa (2002), la falta de una adecuada organización territorial en Colombia es de tipo estructural, pues no ha existido un proyecto territorial nacional ni un propósito explícito de construir el Estado Nación moderno; por ello, lo que ahora se hace es una re-edición de actos irracionales en su configuración territorial, con nuevas formas regionales, bajo la lógica actual de regiones competitivas. Y aunque el proceso de ordenamiento territorial es estructural, lo importante es el desarrollo, pero éste no depende de crear regiones, depende de los logros colectivos para armonizar, avanzar, lograr una imagen objetivo.

La visión de futuro para los municipios se ha impulsado con las leyes 152/1994 y 388/1997 y algunos lo han logrado ha- 
cer con adecuados ejercicios prospectivos, en conjunto con la comunidad; también lo han hecho algunos departamentos con la Ley 152/1994 y áreas metropolitanas, apoyadas por la Ley 1625/2003, pero no existe a nivel nacional, la visión de futuro plasmada en un modelo propio. La región sería el hermano mayor de los municipios y de las áreas metropolitanas, en caso de que éstos necesiten un referente de cobertura superior a sí mismos. Pero este hermano mayor, ċserá más eficiente que los menores?; cla eficiencia regional debería ser aún mejor que la de los departamentos? Y si los departamentos se están debilitando, cen quién recae la responsabilidad del desarrollo cuando tampoco se consolidan las regiones? Necesariamente, regresa al ámbito local, entonces, ċuál sería el sentido de nuevas divisiones intermedias, sin aún falta fortalecer adecuadamente a los municipios?

Pareciese que la orientación hacia una nueva regionalización fuera la respuesta a la falta de acción de aquellas unidades menores que no han tenido una gestión pública eficiente. Si se espera que fuese de esta manera, la regionalización tan solo sería un espejismo para anestesiar intereses, mientras que la Nación no puede dar respuesta con una política estructural y las localidades, en su gran mayoría, esperan que otro haga por ellas, lo que no han "podido" hacer con su propia dinámica. Para Cuervo (2003) es necesario hacer explícito el sentido político y la acción colectiva de la región, ello merece una reflexión ética, teórica y política. Lo que el ordenamiento territorial discute no es si las regiones administrativas corresponden con las funcionales, sino cómo poner el conocimiento al servicio de la convivencia, de un progreso y democracia duradera y legítima.

Para Velásquez (2010), dado que en Colombia los departamentos no cuentan con planes territoriales, se dificulta resolver problemas de escala supralocal, lo que obliga a utilizar otros mecanismos, como los planteados en la LOOT, referidos a regiones (RAP y RGP). Pero si los municipios, las áreas metropolitanas y los departamentos cuentan con herramientas e instrumentos para administrar sus jurisdicciones y manifiestan que no les funcionan, se esperaría que las nuevas regiones contaran con suficientes; sin embargo, no las tienen. Según la CEPAL (2010), las sinergias entre agentes públicos y privados, niveles de gobierno, sectores productivos locales y mercados más amplios, son esenciales para activar el desarrollo territorial. Por ello, las verdaderas políticas de descentralización y de asignación territorial de recursos, deben potenciar el desarrollo de las regiones más rezagadas y atender sus necesidades más apremiantes. En este sentido, se requiere un poco más de claridad sobre dos asuntos: ċlo prioritario para el desarrollo es la región o es la asociación?; cuando se anima a la construcción de región para realizar macroproyectos, cen realidad lo importante son los proyectos regionales?
Respecto a las asociaciones, el DNP (2013a) ha establecido que algunas dejan de lado su objetivo central de promover el desarrollo y se convierten en ejecutores de obras públicas sin visión de futuro, situación que genera dudas sobre los beneficios potenciales del instrumento. Goulet (2008) recomienda promover un enfoque subnacional, que sea fortalecido con fondos, para impulsar sinergias que se autoperpetúen y que realimenten la cooperación. Por ello, la cohesión territorial se logra con proyectos estratégicos movidos desde la base local y que beneficien a un considerable grupo social; no se logra con una sumatoria de entidades territoriales en un mapa. El nuevo mapa de Colombia debe incluir los proyectos regionales, que posibiliten un enlace de circunstancias para que la población se empodere de su territorio, no para que unos pocos se enriquezcan con las ganancias de "macro proyectos regionales".

Rincón (2012) establece que el análisis del tema se debe hacer sobre el proyecto regional, porque resulta más acotado y tiene mayor reconocimiento legislativo en la LOOT; tiene la bondad de poder articular políticas de orden nacional y local, porque actúa como interfaz o conexión para enfrentar problemáticas que rebasan los límites político-administrativos y permiten llevar a cabo un conjunto de actividades coordinadas e interrelacionadas orientadas a cumplir un objetivo específico. Esto con el fin de evitar lo que Revéiz (2013) ha detectado como una falla de la descentralización, el que por razones de clientelismo político y de visión cortoplacista, se ha estado privilegiado el interés en el sistema general de participaciones y de regalías, por encima de los demás sistemas.

Un asunto es que existen nuevas dinámicas mundiales que le exigen a los Estados propiciar procesos de integración regional, teniendo en cuenta los imperativos de la actual coyuntura internacional, en materia de competitividad y crecimiento económico, como lo expresa la AMB (2014) y otro asunto es que internamente en un país, como Colombia, se agudicen los desequilibrios territoriales, en gran parte debido a la ineficiencia en la gestión de las responsabilidades públicas. Si las entidades territoriales existentes no son eficientes en su gestión pública, entonces, lo novedoso no es la reconfiguración regional, sino la eficiente gestión pública y, para ello, no se requiere dividir más el presupuesto nacional, se requiere priorizarlo y optimizarlo, estratégicamente, en el marco de un sistema de desarrollo, que considere un modelo de ordenamiento territorial.

En conclusión, variadas apuestas reivindican la importancia de la reconfiguración regional, adelgazando y engrosando el tema, según la perspectiva de turno, pero es necesario continuar motivando la reflexión constante y más ahora, enlazada con el proceso de ordenamiento territorial. De ahí que surja la inquietud sobre lo que realmente se quiere o debe reconfigurar en Colombia: ¿̇La división territorial, las competencias 
de las nuevas instancias de planeación, la orientación del desarrollo, el proceso de descentralización, la eficiencia de las unidades administrativas, el empoderamiento colectivo o los recursos financieros? Si en Francia, con más de tres décadas de consolidación regional se sintetizan las regiones para optimizar mejor los recursos financieros, que ahora son inclusive menores, en Colombia es el momento de pensar, objetivamente, si la solución es crear un nuevo mapa con regiones y distribuir más el presupuesto o concretar un sistema de desarrollo, que nos permita hacer realidad un modelo de armonía territorial. Más que la región, lo que se requiere en Colombia es el ejercicio de desarrollo colectivo, liderado por instancias superiores, con decisiones y acciones desde el ámbito local, con algunas estrategias que requieren niveles de intervención intermedia, que no necesariamente obligan a la conformación de regiones.

Aunque urge contar con elementos para tomar decisiones desde los territorios locales y los organismos de dirección nacional en pro del desarrollo, la respuesta inmediatista no puede dejársele a la posibilidad de armar otro grupo de instancias de decisión, como expresa Posada (2014); a nivel mundial estamos en la era de las fusiones y requerimos pasar de la planeación individual a la acción colectiva, en el contexto de la realidad que tenemos y que necesitamos; la gestión colectiva dinamiza los procesos de armonización del territorio, no como un instrumento más sino con acciones transformadoras graduales, en un horizonte de largo plazo. Con el espejo de Francia, que teniendo bien definida la región como la principal entidad territorial, ha decidido sintetizarlas para lograr mayor eficiencia en el proceso descentralizador y para optimizar el ordenamiento territorial; se recomienda que en Colombia revisemos los siguientes asuntos, para en las pretensiones de utilizar la figura regional:

- Una real descentralización permite la transferencia de competencias administrativas del gobierno hacia las entidades territoriales locales.

- La concreción del marco normativo de las entidades territoriales evita duplicidad de competencias y de asignación de recursos.

- La valorización del potencial local ayuda a visualizar al país internacionalmente.

- Los grandes ejes del desarrollo deben apuntar a prevenir efectos de las principales tensiones mundiales.

- Es clave privilegiar y promover la asociación y cooperación intermunicipal, incluyendo su financiamiento.

- Así como los SRADDT es necesario considerar planes o esquemas sobre planificación y gestión estratégica intermunicipal, interdepartamental y multinacional, con el fin de fomentar las colectividades territoriales.

- La constitución de regiones con un tamaño de tan solo dos departamentos, no garantiza su continuidad, porque para competir se requieren alianzas más robustas, especialmente, en el ámbito de la competencia internacional.
- Incentivar la transferencia de competencias hacia las diferentes formas de asociación intercomunal, con el fin de que estas figuras puedan ser capaces de ayudar, de manera eficiente, a los municipios, para desarrollar proyectos de envergadura.

- Introducir recompensas políticas y financieras positivas, por el avance en objetivos comunes.

- Vale más una región conformada por la voluntad colectiva de su población, que por un proyecto regional coyuntural, financiado con recursos derivados de la explotación de recursos naturales no renovables.

Agradecimiento: Las autoras agradecen a la U.D.C.A, por financiar el proyecto de investigación "La coherencia de la gestión territorial en Colombia", que dio origen a este manuscrito. Conflicto de Intereses: El manuscrito fue preparado y revisado por las autoras, quienes declaran ser las únicas autoras y no presentan conflicto de intereses.

\section{BIBLIOGRAFÍA}

1. AMB. 2014. RAPE, región central territorio para la paz. Gobernaciones de Cundinamarca, Boyacá, Tolima y Meta. Alcaldía Mayor de Bogotá. Colombia. 185p.

2. ARCHES, J.; AUCLAIR, L.; FORGUE, C. 2013, Les Schémas Régionaux d'Aménagement et de Développement Durable des Territoires: Définition, enjeux. Politech Tours-Génie de l'aménagement. Francia. 76p.

3. BOISER, S. 1998. Teorías y metáforas sobre desarrollo territorial. (Chile). Rev. Austral Ciencias Sociales. 2:518.

4. CARRIZOSA UMAÑA, J.; FALS BORDA, O.; MONCAYO JIMÉNEZ, E.; MENDOZA MORALES, A.; CASTRO OSORIO, S.L.; ESPINOSA RICO, M.A.; BLANCO, J.A. 2002. Regionalización y Ordenamiento Territorial: O la encrucijada del proyecto territorial nacional. Bol. Soc. Geográfica Col. 45:70-85.

5. CEPAL. 2010. Brechas por cerrar, caminos por abrir. Comisión Económica para América Latina y el Caribe. Naciones Unidas. 291 p.

6. CEPAL 2014. Métodos y aplicaciones de la planificación regional y local En América Latina. Serie Desarrollo Territorial 17 Naciones Unidas. 85p.

7. CIEP. 1998. Colombia país de regiones. Centro de Investigación y Educación Popular. Cinep. Colciencias. Colombia. Tomo 4. 405p. 
8. CUERVO GONZÁLEZ, M. 2003. Pensar el territorio: los conceptos de ciudad-global y región en sus orígenes y evolución. Gestión pública. Chile. 60p.

9. DNP. 2013a. Balance preliminar de los procesos asociativos en Colombia. Departamento Nacional de Planeación. Colombia. 45p.

10. DNP. 2013b. Lineamientos para el proceso de ordenamiento territorial departamental. Departamento Nacional de Planeación. Colombia. 45p.

11. DOTSEY, K. 2013. SRADDT: Elaboration et impact sur les politiques régionales, SARDDT Schéma Régional de Développement Durable du Territoire. Francia.

12. ESPEJO MARIN, C. 2003. Anotaciones entorno al concepto de región. Rev. Nimbus (España).11-12: 6787.

13. ESPINOSA, M. 2002. Región: O el retorno del debate sobre la cuestión nacional en los países dependientes. Colombia. 26p. Disponible desde Internet en: www. sogeocol.edu.co (con acceso 14/12/2014).

14. FALS BORDA, O. 1993. El reordenamiento territorial: itinerario de una idea. Universidad Nacional de Colombia. Análisis Político. 20:97-105.

15. FALS BORDA, O. 2000. Acción y espacio: autonomías en la nueva república. Tercer Mundo Editores -Lepri. Colombia. 99p.

16. FONDO MONETARIO INTERNACIONAL -FMI-. 2014. Perspectivas de la economía mundial: Secuelas, nubarrones, incertidumbres. Estados Unidos de América. 267p.

17. GRANDGUILLOT, D. 2014. Les Collectivités Territoriales en France 2014-2015, Editions Lextenso, 3e. Éd. Editeur Gualino. Francia. 48p.

18. GOULET, R. 2008. El Libro Verde sobre la cohesión territorial: el camino a seguir. En: revista INFOREGIO No. 28. Unión europea. 20p. Disponible desde Internet en: http://ec.europa.eu/regional_policy/consultation/terco/index_es.htm (con acceso 05/02/2015).

19. INSTITUTO GEOGRAFICO AGUSTIN CODAZZI, IGAC. 2012. Regiones geográficas (naturales) de Colombia.

20. IMC. 2014. ¿Quién manda aquí? La gobernanza de las ciudades y el territorio en México. 1a edición: sep- tiembre 2014. Instituto Mexicano para Competitividad A.C. 278p.

21. LEY ORGÁNICA DE ORDENAMIENTO TERRITORIAL, LOOT, LEY 1454 DE 2011, (Junio 28), reglamentada por el Decreto Nacional 3680 de 2011, por la cual se dictan normas orgánicas sobre ordenamiento territorial y se modifican otras disposiciones.

22. LNRF. 2014. La Nouvelle République Francia. Réforme territoriale: l'Etat engrangerait 12 à 25 milliards. Vallini André, secrétaire d'Etat. article publié le 9 mai 2014.

23. LOUBET, L. 2013. Lapprentissage de la coopération intercommunale: modalités et instruments, Lespace politique. Revue en ligne, $n^{\circ} 18$. Disponible desde Internet en: http://espacepolitique.revues.org/2454, (con acceso 12/02/2015).

24. MENDOZA MARTELO, G.; CEPEDA AMARÍS, A.; ESTUPIÑÁN ACHURY, L. 2013. Una mirada a las regiones desde la justicia constitucional. Colección Textos de Jurisprudencia. Editorial. Universidad del Rosario. 494p.

25. MIEDES, B.; SÁNCHEZ, C. 2014. Alternativas de economía social para el tránsito socioecológico. De la "conciencia de lugar" a la "inteligencia territorial". Universidad de Huelva. España. 143p.

26. PASQUIER, R. 2012. Le Pouvoir régional, Mobilisations, décentralisation et gouvernance en France, Presses de SciencesPo. Francia. 384p.

27. PINZÓN, M.; ECHEVERRI, I. 2012. La sostenibilidad ambiental regional: una propuesta metodológica para su estudio más allá de la ecología urbana. Universidad Nacional. (Colombia). Luna Azul. 34:131-147.

28. POSADA, A. 2014. De la planeación a la gestión ambiental colectiva: posibilidades en Bogotá - Colombia. Rev. U.D.C.A. Act. \& Div. Cient. 17(1):257-264.

29. RAMÍREZ, T. 2012. Definición de una escala múltiple de Bogotá-región: Una apuesta de sostenibilidad desde la divergencia regional. Procesos de ordenamiento en América Latina y Colombia. Facultad de Artes Universidad Nacional. Colombia. 98p.

30. REYES P., D.; CÓRDOBA HENAO, G. 2012. Los conceptos región y territorio como aporte a los estudios de la lengua. Rev. digital Instituto Caro y Cuervo Lenguas en contacto y bliligüismo. 2: 131-158. Colombia. Disponible desde Internet en: http://www. 
caroycuervo.gov.co/service/revista-digital-lenguasen-contacto, (con acceso 24/04/2015).

31. REVÉIZ, E. 2013. El estado estratega para el ordenamiento territorial. Academia colombiana de ciencias económicas. Colombia. 337p.

32. RINCÓN, M. 2012. Algunas reflexiones en torno al proyecto regional. Procesos de ordenamiento en América Latina y Colombia. Facultad de Artes Universidad Nacional. Colombia. 98p.

33. RODRIGUES, M. 2010. Gobernanza estratégica para el desarrollo: de la experiencia europea a la experiencia latinoamericana. (Venezuela). Rev. CLAD Reforma y Democracia. No. 48. 16p.
34. SÁNCHEZ VALBUENA, H. 2004. Regionalización y ordenamiento territorial: Una propuesta para Colombia. Rev. U.D.C.A. Act. \& Div. Cient. 7(2):3-10.

35. SANTOS, M. 2000. La naturaleza del espacio. Técnica y Tiempo. Razón y Emoción. Ed Ariel. España. 352p.

36. VELÁSQUEZ, F. 2010. La planeación territorial en Colombia: contexto, trayectoria y experiencias. Cuadernos Descentralistas $n^{\circ} 25$. Grupo Propuesta Ciudadana. Primera edición. Perú. 196p.

Recibido: Enero 22 de 2015

Aceptado: Abril 27 de 2015

\section{Cómo citar:}

Posada Arrubla, A.; Berrocal Mendoza, A.E. 2015. Reconfiguracion regional en el marco del ordenamiento territorial; Francia, un espejo para Colombia. Rev. U.D.C.A Act. \& Div. Cient. 18(1): 271-281. 